\title{
MODÉLISATION TRIDIMENSIONNELLE : IDENTIFICATION ET INFLUENCE DU COUPLAGE SUR LES PARAMÈTRES COMPLEXES DES MATÉRIAUX PIÉZOÉLECTRIQUES
}

\section{S. EL NACHEF et M. BRISSAUD*}

INSERM, Unité 281, 151 Cours A. Thomas, F-69424 Lyon cedex 03, France

*INSA, Lab. Génie Electrique et Ferroélectricité, F-69621 Villeurbanne cedex, France

Résumé : La caractérisation des céramiques piézólectriques s'effectue habituellement à partir de modèles unidimensionnels décrits dans les normes IEEE. Les modes sont supposés découplés ce qui n'est pas le cas pratiquement. Le modèle tridimensionnel de vibration d'un disque épais piézoélectrique prend en compte à la fois les couplages élastiques et piézoélectriques ainsi que les pertes mécaniques du matériau. Le but de ce travail est l'utilisation des techniques d'optimisation pour identifier simultanément les résonances radiales et axiales d'un disque céramique. La méthode numérique mise en œuvre est la méthođe du SIMPLEXE qui est bien adaptée au cas où il y a un grand nombre de paramètres à déterminer. Nous nous sommes particulièrement intéressés à montrer l'influence des paramètres de couplage $\mathrm{C}_{12}, \mathrm{C}_{13}$ et h31 sur la forme des courbes de résonance obtenues.

\begin{abstract}
The characterization of piezoceramic materials is carried out by means of one dimensionnal IEEE standards for which the resonance modes are not coupled. The 3D model of the piezoceramic disk vibration modes takes into account both elastic and piezoelectric couplings and also the mechanical losses of the material. The aim of this paper is the use of an optimization technique called the SIMPLEX method for simultaneously identifing axial and radial modes of a thick piezoceramic disk. It is a suitable method when a lot of parameters are to be determined. This paper focuses of the influence of the coupling parameters as $\mathrm{C}_{12}, \mathrm{C}_{13}$ and $\mathrm{h}_{31}$ on the form of the resonance curves which can obtained.
\end{abstract}

\section{I - INTRODUCTION}

La caractérisation des céramiques piézoélectriques s'effectue habituellement à partir de modèles unidimensionnels appelés modèles de MASON [1] et décrits dans les normes IEEE [2]. Ils permettent de déduire les paramètres élastiques, diélectriques et piézó́lectriques des mesures de fréquences de résonances de divers échantillons de formes et de géométries particulières afin de privilégier un mode particulier. On obtient ainsi l'ensemble des coefficients d'un matériau piézoélectrique et les pertes diélectriques et mécaniques sont prises en compte en utilisant des paramètres complexes. Nous avons proposé une nouvelle méthode très différente consistant à minimiser l'écart quadratique existant entre les points expérimentaux et calculés à partir d'un modèle analytique unidimensionnel classique. Nous avons montré les limites d'application de ce modèle [3].

Le but de cet article est l'utilisation des techniques d'optimisation pour identifier simultanément les résonances radiale et axiale d'un disque céramique piézoélectrique au moyen du modèle tridimensionnel que nous avons développé [4] incluant les couplages mécaniques (POISSON) et piézoćlectrique existant dans tous les matériaux. La méthode numérique utilisée pour résoudre ce problème est la méthode du SIMPLEXE souvent mise en cuvre pour l'identification des paramètres des procédés industriels. La principale difficulté avec la méthode d'optimisation est la détermination d'un vecteur de départ fiable assurant une bonne convergence de l'algorithme d'identification. Les résultats issus des méthodes classiques ne réalisant pas cette condition, nous avons imaginé une méthode originale reposant uniquement sur des résultats tridimensionnels. Les résultats obtenus ainsi par la méthode d'optimisation sont en très bon accord avec les résultats expérimentaux. Nous nous sommes intéressés particulièrement à l'influence des paramètres de couplage $C_{12}, C_{13}$ et h31 sur la forme des courbes obtenues.

\section{II - MODÉLISATION ANALYTIOUE}

En supposant que des ondes planes se propagent selon le rayon a et l'épaisseur 2a3 du disque céramique piézoélectrique (figure 1), seules subsistent les contraintes $\left(T_{1}, T_{2}, T_{3}\right)$ et les déformations $\left(S_{1}, S_{2}, S_{3}\right)$ d'extensioncompression. Le disque est alimenté par une différence de potentiel $\emptyset$ appliquée sur les faces métallisées perpendiculaires à l'axe 3 de polarisation. En calculant les charges créées sur ces faces et en intégrant ensuite le champ électrique le long de son épaisseur, on peut déterminer l'expression de l'impédance électrique du disque céramique piézoélectrique. On obtient [4] [6] :

$$
Z=\frac{1}{j C_{0} \omega}\left[1-\frac{h_{31}}{\beta_{33}^{S}} \frac{h_{31} C_{33}^{D}-h_{33} C_{13}^{D}}{C_{33}^{D}-C_{13}^{D^{2}} \alpha J_{o}(\alpha a)} \alpha J_{o}(\alpha a)-\frac{h_{33}}{\beta_{33}^{S}} \frac{J_{a} h_{33}-h_{31} C_{13}^{D} \alpha J_{o}(\alpha a)}{J_{a} C_{33}^{D}-C_{13}^{D^{2}} \alpha J_{o}(\alpha a)} \frac{\operatorname{tg}\left(\alpha_{3} a_{3}\right)}{\alpha_{3} a_{3}}\right]
$$


où $\alpha$ et $\alpha_{3}$ sont les modules des vecteurs d'ondes correspondant aux modes radial et axial, $C_{0}$ est la capacité du disque libre, $\mathrm{h}_{31}, \mathrm{~h}_{33}, \mathrm{C}_{11}^{\mathrm{D}}, \mathrm{C}_{12}^{\mathrm{D}}, \mathrm{C}_{13}^{\mathrm{D}}$ et $\mathrm{C}_{33}^{\mathrm{D}}$ sont respectivement les constantes piézoélectriques et élastiques du matériau. $\mathrm{J}_{0}$ et $\mathrm{J}_{1}$ sont les fonctions de Bessel de première espèce et d'ordres 0 et un. $\mathrm{J}_{\mathrm{a}}$ est reliée à $\mathrm{J}_{0}$ et $\mathrm{J}_{1}$ par la relation

$$
\mathrm{J}_{\mathrm{a}}=\alpha C_{11}^{\mathrm{D}} \mathrm{J}_{\mathrm{o}}(\alpha \mathrm{a})-\left(C_{11}^{\mathrm{D}}-\mathrm{C}_{12}^{\mathrm{D}}\right) \mathrm{J}_{1}(\alpha \mathrm{a}) / \mathrm{a}
$$

Remarquons que les constantes élastiques comportent chacune une partie réelle et une partie imaginaire qui caractérise les pertes dans le matériau soit $C_{i j}^{D}=C_{i j}^{D}\left(1+j p_{i j}\right)$ où pij peut être fonction de la fréquence [6].

Par contre, du fait du mode de vibration libre du disque les pertes diélectriques peuvent être négligées [3] et $\varepsilon_{33}^{S}$ est purement réelle.

\section{III - MÉTHODE DIDENTIFICATION}

La modélisation tridimensionnelle précédente montre qu'il y a douze paramètres à déterminer. Parmi les différentes techniques d'optimisation, la méthode du SIMPLEXE nous a paru la mieux adaptée à notre problème. Cette méthode, habituellement utilisée dans d'autres domaines, consiste à minimiser l'écart quadratique existant entre les valeurs expérimentales et les valeurs calculées à partir d'un modèle analytique. A chaque itération les paramètres du modèle sont modifiés de manière à réduire progressivement cet écart.

Le problème principal des méthodes d'identification est le choix du vecteur initial des parametres qui assurera la convergence de la méthode. Les méthodes de détermination unidimensionnelles ne conduisent pas à un vecteur initial fiable. Cela nous a conduit à développer une méthode originale reposant uniquement sur des résultats issus de la modélisation tridimensionnelle. De plus parmi toutes les résonances qui apparaissent dans les courbes expérimentales, il est nécessaire de reconnaitre celles qui appartiennent effectivement au mode radial et au mode axial.

\section{IV - RÉSULTATS ET DISCUSSIONS}

Le fait de trouver des vecteurs de paramètres pour lesquels un des deux modes de résonance axial ou radial est très peu prononcé, nous a incité à étudier l'influence des paramètres de couplages intervenant dans le modèle tridimensionnel șur l'allure générale de la courbe de l'impédance électrique de la céramique piézoélectrique en fonction de la fréquence.

Partant du vecteur de paramètres optimaux qui donne une identification parfaite des résonances principales (figures 2 et 3) en modes axial et radial, nous modifions un paramètre à la fois parmi les paramètres de couplages mécaniques $C_{12}^{D}$ et $C_{13}^{D}$ et piézoélectrique $h_{31}$, en le multipliant par un coefficient correctif. Ensuite, nous traçons la courbe théorique avec le nouveau jeu de paramètres et nous la comparons à la courbe expérimentale.

Chacun de ces paramètres est un nombre complexe dont nous avons identifié les parties réelle et imaginaire, ainsi :

$$
C_{12}^{D}=R_{e}\left(C_{12}^{D}\right)+j I_{m}\left(C_{12}^{D}\right) \quad C_{13}^{D}=R_{e}\left(C_{13}^{D}\right)+j I_{m}\left(C_{13}^{D}\right) \quad h_{31}=R_{e}\left(h_{31}\right)+j I_{m}\left(h_{31}\right)
$$

L'influence sur la courbe de la partie imaginaire des paramètres de couplage mécanique est très peu significative [7]. Nous limiterons donc notre étude au rôle de leurs parties réelles en les multipliant d'abord par des coefficients de plus en plus petits puis de plus en plus grands. Nous observons et nous analysons l'influence de ces multiplications sur les résonances principales axiale et radiale.

INFLUENCE DE $\mathrm{C}_{12}^{\mathrm{D}}$

a - Multiplication par un coefficient $<1$

* Influence sur la résonance radiale : l'amplitude du pic diminue considérablement et la fréquence de résonance se décale très rapidement vers les basses fréquences.

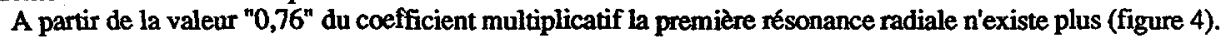

* Influence sur la résonance axiale : le pic reste très bien identifié aussi bien en fréquence qu'en amplitude même si $R_{e}\left(C_{12}^{D}\right)$ s'annule.

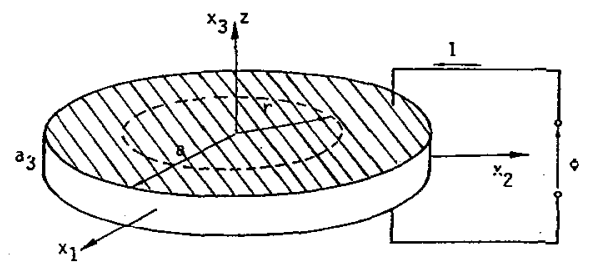

Fig. 1 - Définitions et géométrie 
b - Multiplication par un coefficient $>1$

* Influence sur la résonance radiale : l'amplitude du pic diminue très rapidement et d'une façon régulière et la fréquence de résonance se décale vers les hautes fréquences (figure 5).

* Influence sur la résonance axiale : le pic reste très bien identifié.

Il est à noter que quelle que soit la valeur de $\mathrm{C}_{12}^{\mathrm{D}}$ le pic de résonance axiale est correctement identifié.

INFLUENCE DE $\mathrm{C}_{13}^{\mathrm{D}}$

a - Multiplication par un coefficient $<1$

* Influence sur la résonance radiale : l'amplitude du pic diminue très rapidement et la fréquence de résonance se décale lentement vers les hautes frequences.

A partir de la valeur " 0,5 " du coefficient multiplicatif, la résonance devient très peu prononcée (figure 6).

* Influence sur la résonance axiale : l'amplitude du pic diminue, mais beaucoup plus lentement que celle du pic radial et la fréquence de résonance resté inchangée.

b - Multiplication par un coefficient $>1$

* Influence sur la résonance radiale : l'amplitude du pic diminue et la fréquence de résonance se décale très rapidement vers les basses fréquences, beaucoup plus rapidement que lors de la multiplication de $R_{e}\left(C_{12}^{D}\right)$ par un coefficient $>1$.

A partir du coefficient multiplicatif "1.06" la première résonance radiale n'existe plus (figure 7).

* Influence sur la résonance axiale : l'amplitude du pic augmente considérablement, elle double lorsqu'on multiplie $\mathrm{R}_{\mathbf{e}}\left(\mathrm{C}_{13}^{\mathrm{D}}\right)$ par "1.06" et la fréquence de résonance reste inchangée.

\section{INFLUENCE DE h31}

h31 est le coefficient de couplage piézoélectrique entre les directions radiale " 1 " et axiale " 3 ". Le coefficient multiplicatif est appliqué sur les parties réelle et imaginaire de $h_{31}$.

$h_{31}$ n'a pas d'influence sur les fréquences de résonance [7].

a - Multiplication par un coefficient $<1$

* Influence sur la résonance radiale : l'amplitude du pic diminue lentement.

Même si on multiplie h31 par zéro, on n'élimine aucun des pics de résonances radiales (figure 8).

* Influence sur la résonance axiale : l'amplitude du pic diminue lentement.

Même si on multiplie h31 par zéro, on n'élimine aucun des pics de résonances axiales (figure 9).

\section{b - Multiplication par un coefficient $>1$}

* Influence sur la résonance radiale : l'amplitude du pic augmente.

* Influence sur la résonance axiale : l'amplitude du pic augmente.

\section{$\checkmark-$ CONCLUSION}

Cette méthode nous a montré línfluence de chacun des paramètres de couplage élastique et piézoélectrique sur la fréquence de résonance et l'amplitude des modes axial et radial principaux. Nous avons observé que pour certaines valeurs de $C_{12}^{D}$ et $C_{13}^{D}$, la première résonance radiale disparaissait.

Alors que $C_{12}^{D}$ n'a aucune influence sur la résonance axiale, $C_{13}^{D}$ n'affecte que l'amplitude de la résonance axiale, dans les deux cas la fréquence de rêsonance axiale reste inchangée.

Par contre on ne peut pas trouver une valeur de $\mathrm{h} 31$ qui annule le mode radial.

\section{BIBLIOGRAPHIE}

1 D. BERLINCOURT et al. In "Physical Acoustics". Vol 1A. Academic Press, New York (1964).

2 IEEE Standards on piezoelectricity. SU 31, 2, March 1984 et ANSI IEEE Std 176 (1987).

3 S. EL NACHEF, M. BRISSAUD, D. NOTERMAN. Identification tridimensionnelle des paramètres complexes des disques céramiques piézoélectriques. ACUSTICA (à paraître).

4 M. BRISSAUD. New procedure for piezoceramic characterization. Acoustic Letters, Vol. 13, $\mathrm{n}^{\circ} 10$ (1990).

5 MJ. BOX, D. DAVIES, W.H. SWANN. Techniques d'optimisation non-linéaires. Math. et Stat. pour I'industrie, $\mathrm{n}^{\circ} 5$, I.C.I. (1971).

6. S. EL NACHEF, M. BRISSAUD, D. NOTERMAN. Three dimensionnal characterization of complex parameters of piezoceramic disks using an identification technique. Ultrasonic International 91. Le Touquet (July 1991).

7 S. EL NACHEF. Identification des paramètres complexes des céramiques piézoélectriques par une modélisation tridimensionnelle. Mémoire de DEA. INSA Lyon 1989. 


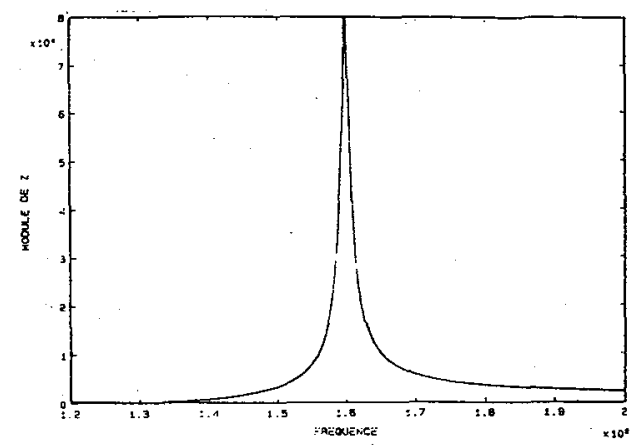

Fig. 2 - Identification simultanée : mode radial

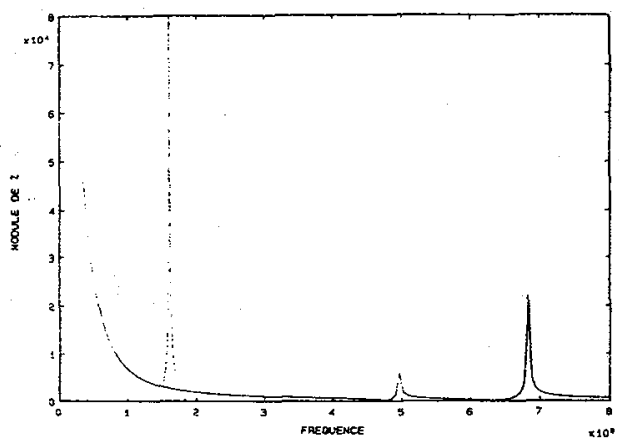

Fig. 4 - Influence de $C_{12}^{D}$ : coefficient multiplicatif 0,75

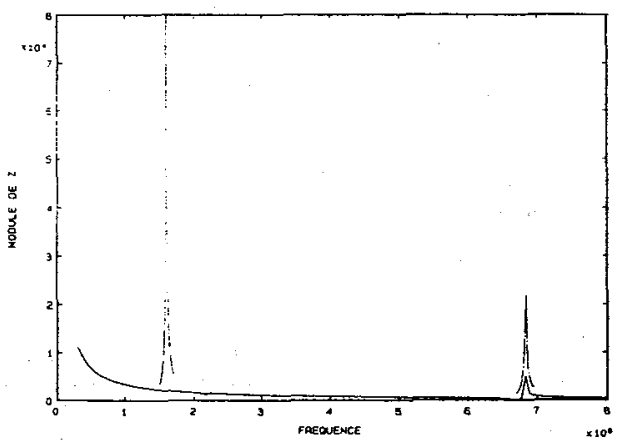

Fig. 6 - Influence de $C_{13}^{D}$ : coefficient multiplicatif 0,5

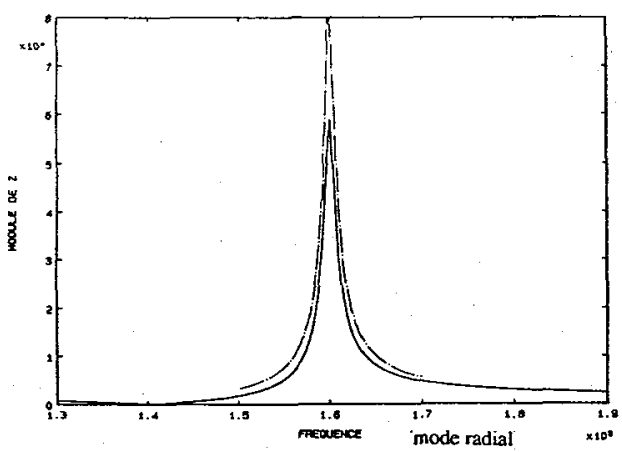

Fig. 8 - Influence de $h_{31}$ : coefficient multiplicatif 0,5

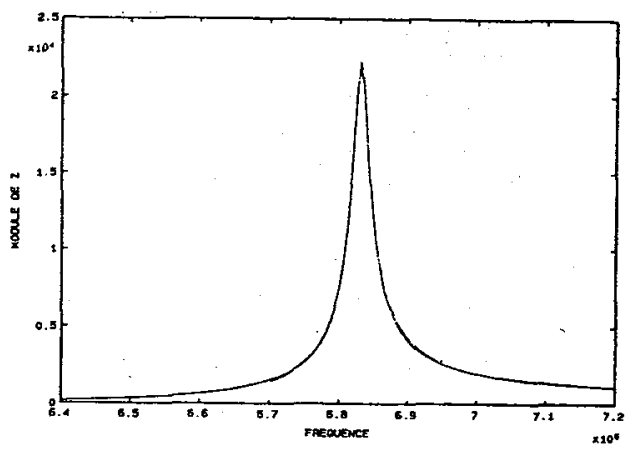

Fig. 3 - Identification simultanée : mode axial

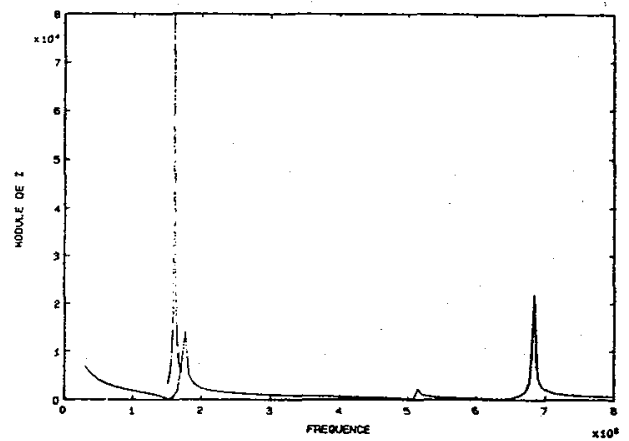

Fig. 5 - Influence de $C_{12}^{D}$ : coefficient multiplicatif 1,05

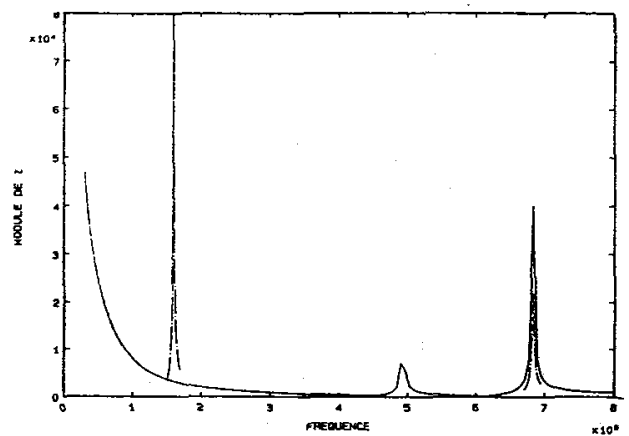

Fig. 7 - Influence de $\mathrm{C}_{13}^{\mathrm{D}}$ : coefficient multiplicatif 1,06

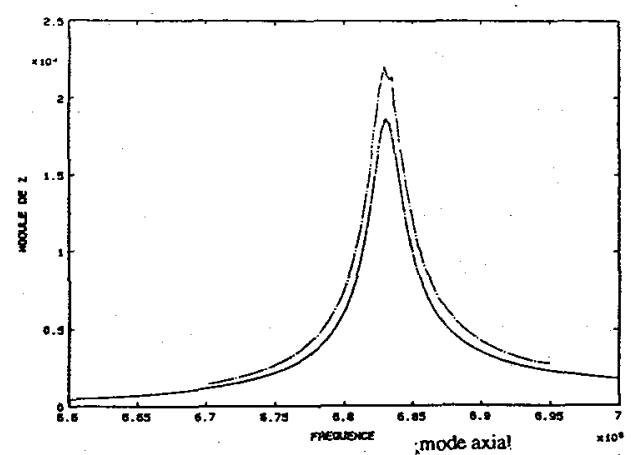

Fig. 9 - Influence de $h_{31}$ : coefficient multiplicatif 0,5 